\title{
Synchronous tunable wavelength spacing dual-wavelength SOA fiber ring laser using Fiber Bragg grating pair in a hybrid tuning package
}

\author{
S.S. Chong, H. Ahmad, M.Z. Zulkifli * A.A. Latif, W.Y. Chong, S.W. Harun
}

\section{A R T I C L E I N F O}

\section{Article history}

Received 15 November 2010

Received in revised form 27 July 2011

Accepted 18 October 2011

Available online 7 November 2011

\section{Keywords:}

Dual-wavelength fiber laser

Fiber Bragg gratings

Semiconductor optical amplifier

\begin{abstract}
A B S T R A C T
A Dual-Wavelength Semiconductor Optical Amplifier (DW-SOA) based fiber ring laser with synchronous wavelength tunability is proposed and experimentally demonstrated. The SOA gain medium strongly suppresses mode competition, thus allowing stable dual-wavelength laser oscillation. The wavelength spacing of the two lasers can be tuned synchronously using a modified hybrid-tuning package incorporating a pair of Fiber Bragg Gratings (FBGs). The DW-SOA demonstrates a laser output with a wavelength spacing of between 0.10 and $8.30 \mathrm{~nm}$ (wavelength shift inequality of 0.08 to $0.75 \mathrm{~nm}$ ). The relationship between the applied strain and wavelength shift of the two tuning modes is also analyzed.
\end{abstract}

(c) 2011 Elsevier B.V. All rights reserved.

\section{Introduction}

Since the first tunable Fiber Bragg Grating (FBG) using mechanical stress was demonstrated [1], the tunability of FBG has seen a tremendous amount of applications in various fields, including potential applications in tunable fiber laser sources, fiber sensors, and Wavelength Division Multiplexing (WDM) optical communication systems [2-4]. Recently, Single-Longitudinal Mode (SLM) fiber lasers capable of generating dual wavelength outputs with tunable wavelength spacing have attracted considerable interest in research and development, especially for the photonics generation of microwave signals [5]. Numerous efforts in developing tunable dualwavelength fiber lasers have been proposed and demonstrated [5-11], and these systems are able to generate a dual-wavelength output with channel spacings of as narrow as $27 \mathrm{pm}$ [9] to as wide as $15 \mathrm{~nm}$ [10]. The use of separate FBGs to generate a dualwavelength SLM output from a fiber laser has also been demonstrated [11], with the output having tunable wavelength spacing between 0.1 and $1.3 \mathrm{~nm}$.

While the progress into dual-wavelength fiber lasers is substantial, issues on the stability and power of the dual-wavelength output still arise. Typically, most fiber lasers utilize Erbium Doped Fibers (EDFs) as the gain medium; primarily because it is a well-known technology that is highly compatible with current fiber optic systems and also inexpensive. However, EDFs suffer from homogeneous line broadening and cross-gain saturation that in turn leads to mode competition and subsequently prevents the generation of the dualwavelength output. In this regard, efforts have now focused on Semiconductor Optical Amplifiers (SOAs) as the gain medium due to its

\footnotetext{
* Corresponding author. Tel.: +60 3 79677133; fax: +60 379674290. E-mail address: mohdzamani@um.edu.my (M.Z. Zulkifli).
}

inhomogeneous broadening effect that eliminates the problem of mode competition $[5,8]$. Consequently, it is a more desirable candidate than the EDF for the generation of stable dual-wavelength output from fiber lasers.

In this paper, we propose and demonstrate a dual-wavelength SOA based fiber laser with the capability to conduct synchronous tuning of the channel spacing. The proposed system utilizes an SOA as the gain medium and a pair of FBGs to provide tunability in the channel spacing. The wavelength spacing can be tuned synchronously from $0.10 \mathrm{~nm}$ to $8.30 \mathrm{~nm}$ (with a wavelength shift inequality of 0.08 to $0.75 \mathrm{~nm}$ ) and produces a stable dual-wavelength output. This system has a significant potential in the photonic generation of microwave signals in the $13 \mathrm{GHz}$ to $1 \mathrm{THz}$ frequency range.

\section{Theory}

The tuning of the channel spacing in this work is accomplished via the application of strain to the FBGs based on a lateral bending beam technique utilizing a hybrid material substrate [12]. The wavelength shift due to the axial strain applied on the FBGs is given as [12]:

$\Delta \lambda=\left(1-\rho_{e}\right) \varepsilon_{z} \lambda_{B}$

where $\rho_{e}=0.22$ is the photo-elastic coefficient, $\varepsilon_{z}$ is the applied strain in the $\mathrm{z}$-direction and $\lambda_{B}$ is the Bragg resonance wavelength. The two FBGs are mounted on both sides of the hybrid material so that one experiences compressive strain while the other experiences tensile strain when mechanical bending is applied to the material (note that the strains are experienced simultaneously, thus giving the synchronous tuning capabilities). Fig. 1 shows illustration of the synchronous FBG pair tuning setup. 


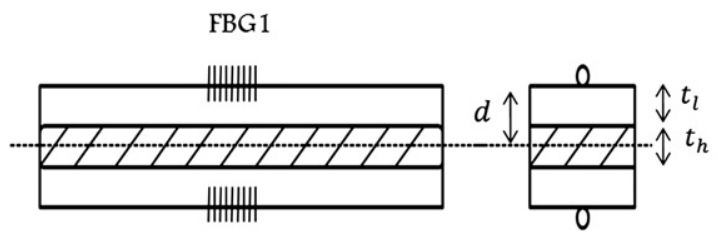

FBG2

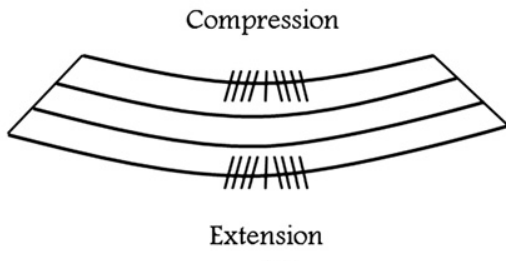

(b)

(a)

Perspex (low Young modulus)

$\square$ Spring steel (High Young modulus)

Fig. 1. Schematic of the (a) hybrid material with both sides mounted by FBG and (b) FBGs under mechanical bending.

(a)

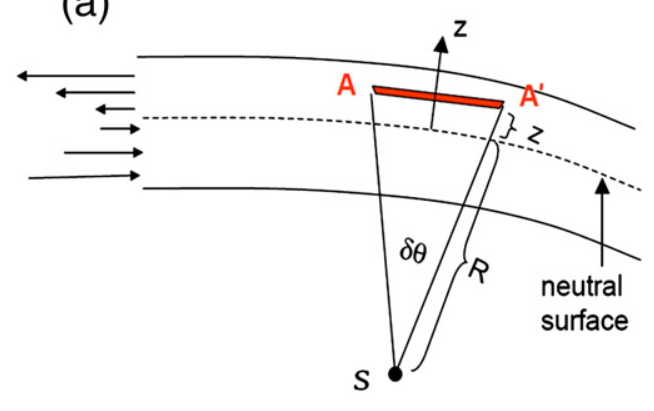

(b)

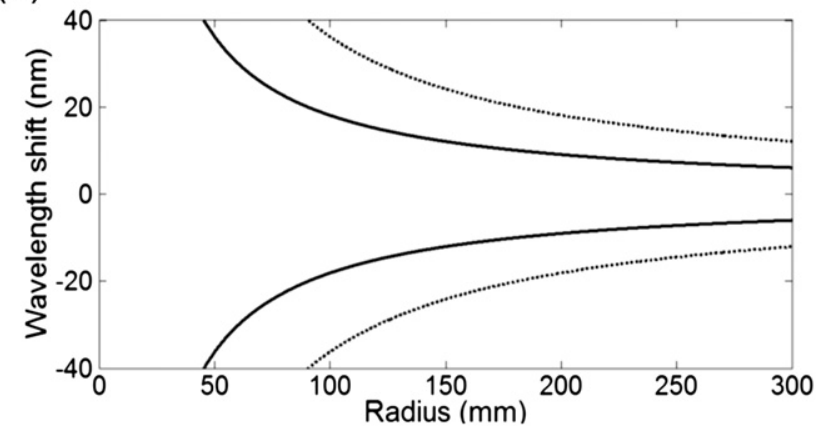

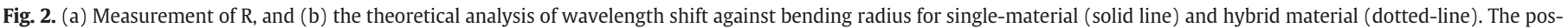
itive wavelength shift accounts for the tensile mode while the negative value accounts for the compression mode.

The induced strain $\varepsilon_{z}$,experienced by FBGs is estimated by the strain-displacement equation [12], whereby:

$\varepsilon_{z}(R)= \pm \frac{d}{R}$

where $R$ denotes the bending radius of the substrate. The measurement of $R$ is shown in Fig. 2(a). A reference point, A is chosen on the neutral surface of the beam when it is in the idle position, and the distance of $A$ to a constant point, $S$ is obtained. When stress is applied to the beam, the beam bends and $A$ shifts to a new position $A^{\prime}$. The distance of $\mathrm{A}^{\prime}$ to $\mathrm{S}$ is $\mathrm{R}$, with $\delta \theta$ being the sector angle. The positive sign and negative sign indicate the tensile and compressive modes respectively. The $d$ factor of the hybrid substrate can be approximated as [12]:

$d=t_{l}+\frac{E_{h} t_{h}^{2}-E_{l} t_{l}^{2}}{2\left(E_{h} t_{h}+E_{l} t_{l}\right)}$

where $E_{l}$ and $E_{h}$ are the Young's modulus of the Perspex and spring steel respectively. In this case, $E_{l}=3.2 \mathrm{GPa}, E_{h}=200 \mathrm{GPa}$, $t_{l}=3 \mathrm{~mm}$ and $t_{h}=1 \mathrm{~mm}$. The relationship between the wavelength shift of FBG and the bending radius of the hybrid material is calculated from Eqs. (1) and (2). With the value of $d$ defined in Eq. (3), the relation produces the result as shown in Fig. 2(b).

Using the proposed configuration, the dual-wavelength output of the SOA-based fiber laser can be tuned in such a way that both channels experience equal shift in the wavelengths in opposite directions, therefore increasing or decreasing the channel spacing as needed.

\section{Experimental setup}

Fig. 3 shows the experimental setup of the proposed SOA based fiber laser. The proposed laser utilizes an SOA as the gain medium and two Apodized Fiber Bragg Gratings (AFBGs), designated AFBG1 and AFBG2 in Fig. 3 to provide the synchronous tuning capability. The 3-dB bandwidth, reflectivity and center wavelength of AFBG1 and AFBG2 are $0.158 \mathrm{~nm}, 60 \%, 1550.920 \mathrm{~nm}$ and $0.154 \mathrm{~nm}, 55 \%$, $1550.950 \mathrm{~nm}$ respectively. The two AFBGs are mounted on both sides of the hybrid material using an adhesive glue which is UV cured for 10 min followed by heat treatment in an oven for $12 \mathrm{~h}$ at $50{ }^{\circ} \mathrm{C}$ to ensure that the glue sets and properly secures the AFBGs to the hybrid material.

The SOA is driven at a current of $350 \mathrm{~mA}$ to achieve maximum signal amplification. An Optical Isolator (ISO) is placed before the SOA to ensure unidirectional propagation of light in the ring configuration, while a Polarization Controller (PC) is used to align the polarization state of the light after passing through the SOA. The output power is extracted using a 90:10 Optical Coupler (OC), with 10\% being taken as the output power for further analysis. The laser output is analyzed using an Optical Spectrum Analyzer (OSA) with a resolution of $0.02 \mathrm{~nm}$. A circulator is also used to route the reflected signals from

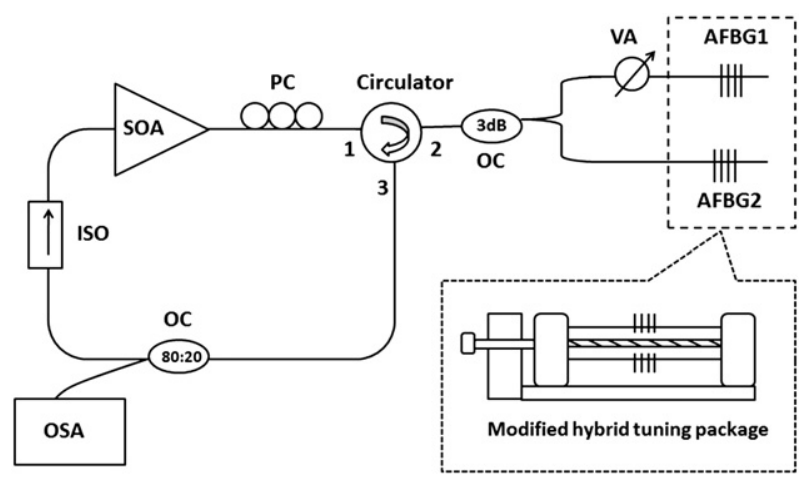

Fig. 3. Schematic of the proposed dual-wavelength SOA fiber ring laser with synchronous wavelength tuning capabilities. 


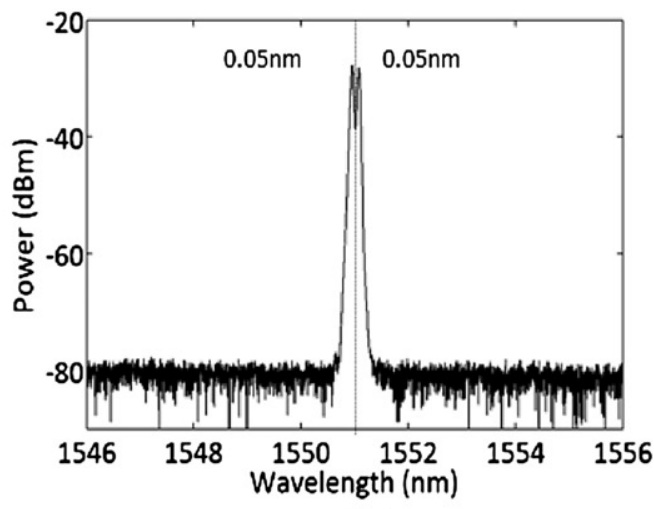

(a)

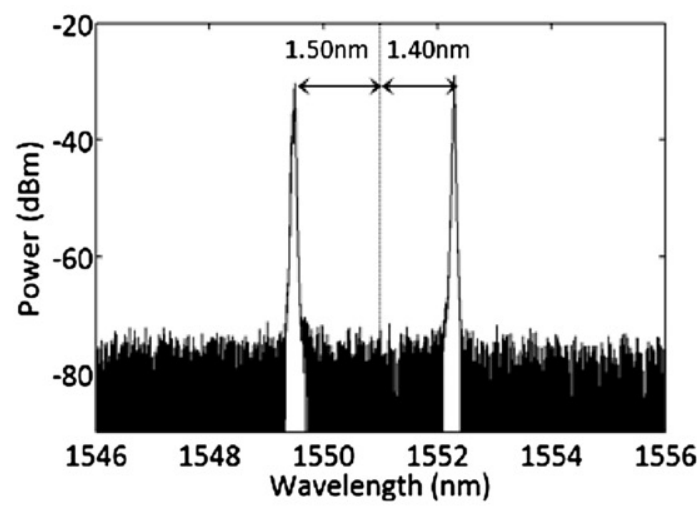

(c)

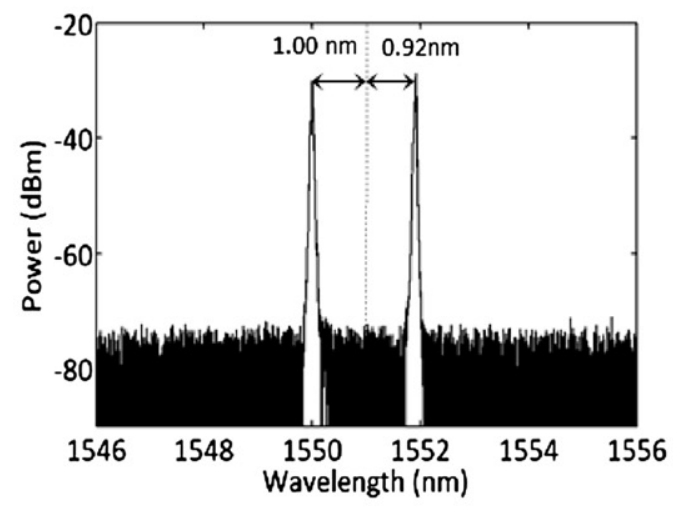

(b)

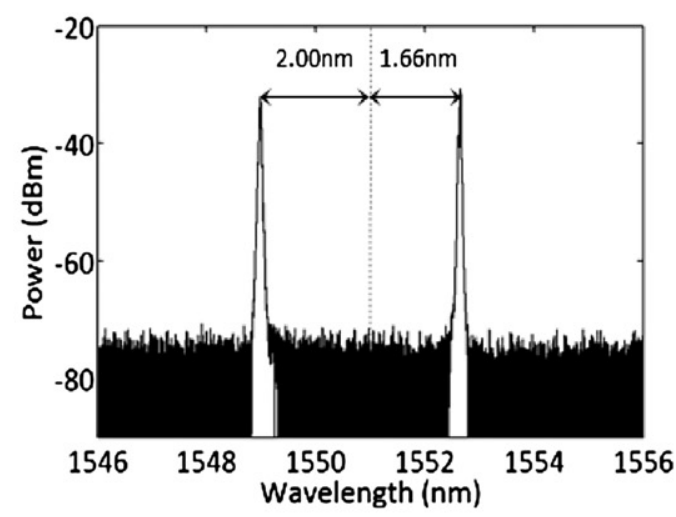

(d)

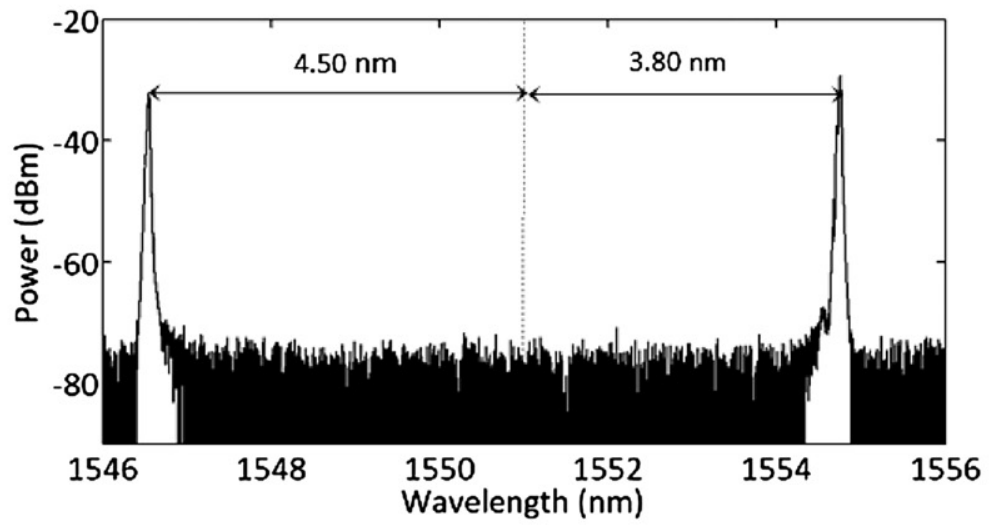

(e)

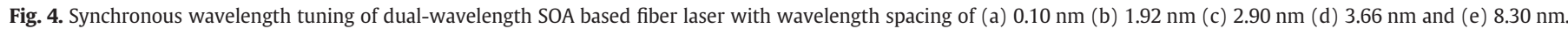

the AFBGs back into the ring lasing. At port 2 of the optical circulator, the optical signal is equally split by a $3-\mathrm{dB}$ optical coupler and reflected by the two AFBGs. When mechanical stress is applied to the hybrid material, AFBG1 experiences compressive strain while AFBG2 experiences tensile strain which lead to the blue (shorter wavelength) and red (longer wavelength) shifts of the Bragg wavelength respectively. A Variable Attenuator (VA) is inserted between the 3-dB optical coupler and AFBG1 to compensate for the difference in reflectivity between the two AFBGs. All free terminations of the system are immersed in index-matching gel to minimize back reflections.

\section{Experimental results and discussion}

Fig. 4 shows the synchronous tuning of the dual-wavelength output in the proposed fiber laser when a bending moment is applied at both ends of the material. By applying displacement to the z-axis of the hybrid material via a gear mechanism, various channel spacings are realized from the dual-wavelength fiber ring laser as the Bragg wavelengths of both AFBGs are observed to shift in opposite directions (depending on whether a tensile or compression strain is applied), thus increasing or decreasing the wavelength spacing between them. 


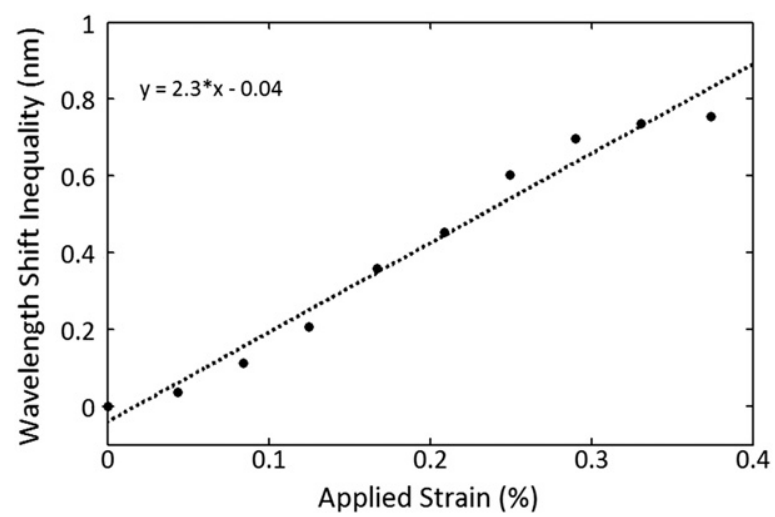

Fig. 5. Wavelength shift inequality (nm) against applied strain (\%).

From Fig. 4(b) to (e), it can be seen that the shift of the wavelengths in both directions agrees as described by the theory, however this shift is not at a magnitude as predicted by the theoretical analysis. This is attributed to the difference in the extension and compression strength that the silica glass is capable of sustaining. The wavelength shift of the two AFBGs in opposite directions is not the same. For instance, as shown in Fig. 4(c), the shift toward the left is $1.50 \mathrm{~nm}$ whereas the shift toward the right is only $1.40 \mathrm{~nm}$. This gives a 'wavelength shift inequality' of about $0.10 \mathrm{~nm}$. In the case of Fig. 4(a), the shift is similar for AFBGs, having a value of about $0.05 \mathrm{~nm}$ in opposite directions with an applied strain of $\pm 0.004 \%$. There is a possibility that this shift may not be symmetrical as can be inferred from Fig. 4(b) to (e). It may look similar which could be due to the limited resolution of the OSA.

Fig. 5 shows the relationship between wavelength shift inequality against applied strain when the modified hybrid tuning package which consist of two FBGs is subjected to applied force. One of the FBGs will experience tensile stress, and the other compression stress.

Fig. 5 shows a linear relationship between the wavelength shift inequality and the applied strain, with a gradient of 2.3 obtained when a trend line is fitted to the graph. This indicates that the wavelength shift inequality approximately doubles as the applied strain is increased. However, above an applied strain of about $0.29 \%$, the FBG becomes deformed, and thus the relationship is no longer linear and instead begins to plateau off. The uncertainty of the coefficient for the linear fit of the curve is about $0.45 \mathrm{~nm} / \%$.

Fig. 6(a) presents a 3-D scan of the dual-wavelength SOA based fiber ring laser with a wavelength separation of $0.10 \mathrm{~nm}$ over a period of $30 \mathrm{~min}$, which indicates its stability with time.

It can be seen that there is a slight fluctuation as observed in both of the laser peaks. The maximum peak power fluctuations are about $1.00 \mathrm{db}$ for both of the lasing peaks. Fig. 6(b) provides a better

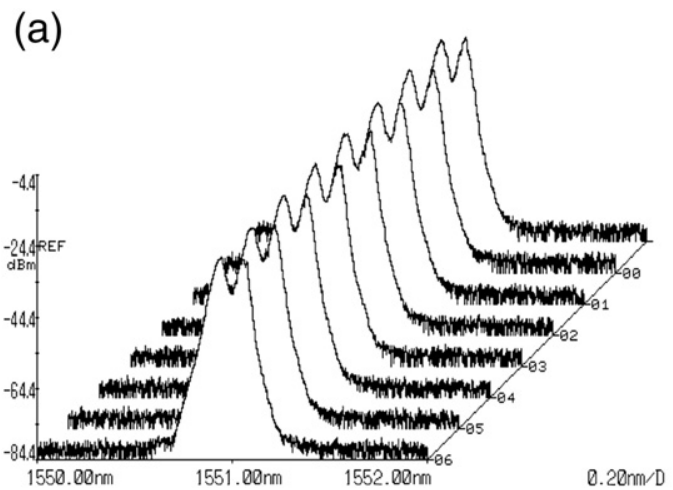

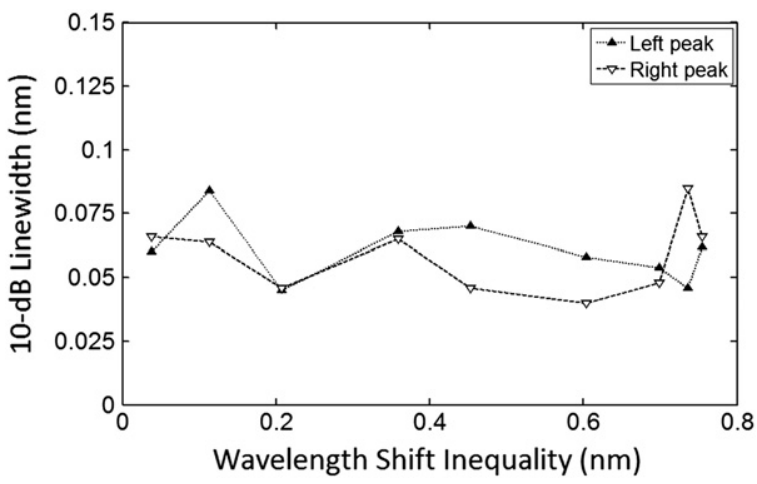

Fig. 7. Variations of $10-\mathrm{dB}$ bandwidth of the dual-wavelength SOA based fiber ring laser against the wavelength spacing. Left lasing peak (dotted-line), right lasing peak (solidline).

representation of the peak power fluctuations over time. One of the ways to increase the stability of the dual-wavelength output is to isolate the setup from external vibrations and to operate in a constant temperature environment. Another approach is to have fine adjustments of the PC. The measurement of other dual-wavelength outputs with wider separations also shows a better stability, which is normally the case.

Fig. 7 shows the variation of the $10 \mathrm{~dB}$ spectral bandwidth for different wavelength spacings against the wavelength shift inequality.

As can be seen from the figure, the measured $10 \mathrm{~dB}$ linewidth of the two lasers outputs remains less than $0.05 \mathrm{~nm}$ for a wavelength shift inequality range from 0.08 until about $0.75 \mathrm{~nm}$, which corresponds to the spacing of the two wavelengths from 0.10 to $8.30 \mathrm{~nm}$. The term 'wavelength shift inequality' has been explained earlier.

\section{Conclusion}

In this work, we propose and demonstrate a dual-wavelength fiber laser utilizing an SOA as a gain medium and with the ability to adjust the channel spacing by the synchronous tuning of the two laser wavelengths. The proposed fiber laser has a tuning range of $0.10-8.30 \mathrm{~nm}$ (wavelength shift inequality from 0.08 to $0.75 \mathrm{~nm}$ ) and the SOA gain medium suppresses the high mode competitions to provide a stable dual-wavelength laser output. A pair of FBGs is incorporated onto a modified tuning mechanism to perform synchronous wavelength spacing tuning, where wavelength shifts in opposite direction are carried out simultaneously. The relationship between the applied strain and difference in wavelength shift is formalized.

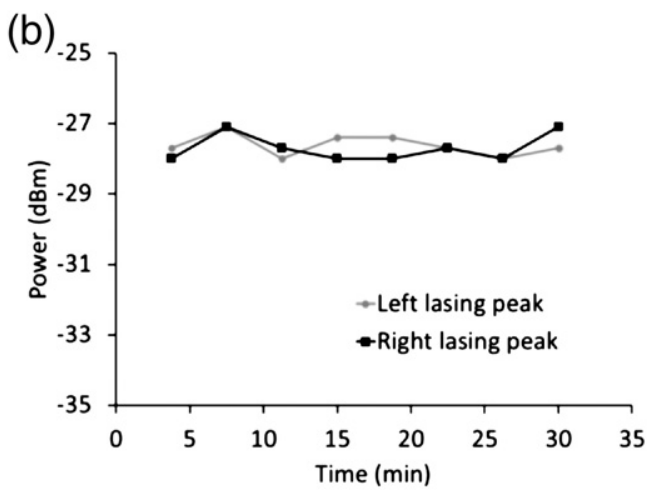

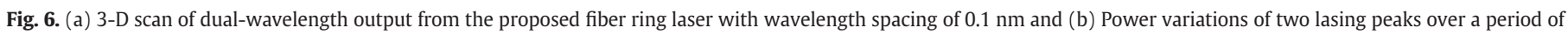
$30 \mathrm{~min}$. 


\section{Acknowledgments}

This project is funded by the University of Malaya, Research University Grant and also T. Kavintheran for editing this work.

\section{References}

[1] A. Iocco, H.G. Limberger, R.P. Salathe, Proc. ECOC, 98, 1998, 1.

[2] Y.W. Song, S.A. Havstad, D. Starodubov, Y. Xie, A.E. Willner, J. Feinberg, IEEE Photonics Technology Letters 13 (11) (Nov. 2001) 1167.

[3] Y.J. Rao, Optics and Lasers in Engineering 31 (1999) 297.

[4] J.A.R. Williams, I. Bennion, Journal of Lightwave Technology 17 (July 1999) 1217.
[5] X.F. Chen, Z.C. Deng, J.P. Yao, IEEE Transactions on Microwave Theory and Techniques 54 (2) (2006) 804.

[6] G.E. Villanueva, P. Perez-Millan, J. Palaci, J.L. Cruz, M.V. Andres, J. Marti, IEEE Photonics Technology Letters 22 (4) (Jan. 2010) 254

[7] X.Y. He, X. Fang, C.G. Liao, D.N. Wang, J.Q. Sun, Optics Express 17 (24) (2009) 21773.

[8] S.L. Pan, X.F. Zhao, C.Y. Lou, Optics Letters 8 (2008) 764.

[9] Y. Y, X.F. Chen, Y.T. Dai, S.Z. Xie, IEEE Photonics Technology Letters 18 (1) (Jan. 2006) 187.

[10] J.R. Qian, J. Su, L. Hong, Optics Communication 281 (2008) 4432.

[11] Y. Wei, B. Sun, Fiber optics 19 (6) (2009) 1252.

[12] C.S. Goh, S.Y. Set, K. Kikuchi, IEEE Photonics Technology Letters 14 (9) (Sept. 2002) 1306. 